\title{
AN OPERATOR STILL NOT SATISFYING LOMONOSOV'S HYPOTHESIS
}

\author{
DON HADWIN \\ (Communicated by Palle E. T. Jorgensen) \\ Dedicated to A. J. Michaels
}

\begin{abstract}
It was shown that quasianalytic unilateral shifts do not satisfy the hypothesis of a famous invariant subspace theorem of $\mathrm{V}$. Lomonosov. We prove that these same shifts do not satisfy the hypothesis of Lomonosov's recent improvement of his theorem.
\end{abstract}

In 1973 Victor Lomonosov revolutionized Hilbert space operator theory when he proved his famous invariant subspace theorem [L1], which includes the following:

If $T$ is an operator commuting with a non-scalar operator $A$, and if $A$ commutes with a non-zero compact operator $K$, then $T$ has a non-trivial (closed) invariant subspace.

For a while operator-theorists wondered if every Hilbert space operator satisfied the hypothesis of Lomonosov's theorem. The unilateral shift was generally suspected to be a counterexample, but C. Cowen [C] proved that the shift does satisfy Lomonosov's hypothesis. Later a counterexample was found; it was proved in [HNRR] that every so-called quasianalytic unilateral weighted shift is a counterexample.

Recently Lomonosov has improved his original theorem; the new theorem includes the following extension of the above result:

If $T$ is an operator commuting with a non-scalar operator $A$, and if there is a net $\left\{B_{\lambda}\right\}$ of operators commuting with $A$ such that

(a) $\left\{B_{\lambda}\right\}$ is weak-operator convergent to a non-zero operator $B$,

(b) $\left\|B_{\lambda}\right\|_{e} \rightarrow 0$ ( \|\|$_{e}$ denotes the essential norm),

then $T$ has a non-trivial closed invariant subspace.

It is the purpose of this note to prove that quasianalytic unilateral weighted shifts do not satisfy the hypothesis of Lomonosov's improved theorem.

Since our proof is a modification of the one in [HNRR], we shall freely use results and notation from that paper. The key additional fact needed is the following elementary lemma. Here $\sigma_{p}(S)$ denotes the point spectrum of the operator $S$.

Received by the editors February 11, 1994 and, in revised form, March 10, 1994.

1991 Mathematics Subject Classification. Primary 47A15. 
Lemma 1. If $\lambda$ is a limit point of $\sigma_{p}(S)$ for some operator $S$, then $|\lambda| \leq\|S\|_{e}$.

Proof. We could appeal to general results in spectral theory that state that there are at most countably many eigenvalues (necessarily isolated) for $S$ that have modulus greater than $\|S\|_{e}$ and that the only limit points of this set are contained in the essential spectrum of $S$ and have modulus at most $\|S\|_{e}$.

However, we can give an elementary Hilbert space proof. Suppose $\lambda_{1}, \lambda_{2}, \ldots$ are distinct eigenvalues for $S$ and $\lambda_{n} \rightarrow \lambda$. For each $n \geq 1$, let $x_{n}$ be a nonzero eigenvector for the eigenvalue $\lambda_{n}$. It follows that $\left\{x_{1}, x_{2}, \ldots\right\}$ is linearly independent. Let $\left\{e_{n}\right\}$ be the orthonormal sequence obtained by applying the Gram-Schmidt process to the $x_{n}$ 's. It follows that $\left(S e_{n}, e_{n}\right)=\lambda_{n}$ for $n=$ $1,2, \ldots$. If $K$ is any compact operator, we have $|\lambda|=\lim _{n \rightarrow \infty}\left|\left(S e_{n}, e_{n}\right)\right|=$ $\lim _{n \rightarrow \infty}\left|\left((S-K) e_{n}, e_{n}\right)\right| \leq\|S-K\|$. Since $K$ is arbitrary, $|\lambda| \leq\|S\|_{e}$.

We state our assertion of a counterexample in a positive form.

Theorem. Suppose $T$ is a quasianalytic unilateral weighted shift operator, $A$ is a non-scalar operator commuting with $T$, and $\left\{B_{\lambda}\right\}$ is a net of operators commuting with $A$ such that $\left\|B_{\lambda}\right\|_{e} \rightarrow 0$ and such that $B_{\lambda} \rightarrow B$ in the weak operator topology. Then $B=0$.

Proof. As in [HNRR] we write $T$ as multiplication by $z\left(T=M_{z}\right)$ on the weighted Hardy space $H^{2}(\beta)$ and we write $A=M_{\varphi}$ with $\varphi$ in the algebra of multipliers $H^{\infty}(\beta)$.

For each $z$ in the closed unit disk, let $k_{z} \in H^{2}(\beta)$ be the unique vector such that $h(z)=\left(h, k_{z}\right)$ for every $h$ in $H^{2}(\beta)$. If $|z| \leq 1$, we define $\mathscr{N}_{z}=$ $\operatorname{ker}(A-\varphi(z))^{*}$, and we let $P_{z}$ denote the projection of $H^{2}(\beta)$ onto $\mathscr{N}_{z}$.

Let $D=\{z:|z|<1\}$ and $\Gamma=\{z:|z|=1\}$. As observed in [HNRR, p. 412], $\varphi(\Gamma)$ is a compact set with area measure zero, and since $\varphi(D)$ is a non-empty open set, we have that $\{z \in D: \varphi(z) \notin \varphi(\Gamma)\}$ is a non-empty open set. The set $\left\{z \in D: \varphi^{\prime}(z)=0\right\}$ is a countable set that clusters at $\Gamma$; thus $F=\left\{z:|z|<1, \varphi(z) \notin \varphi(\Gamma), \varphi^{\prime}(z) \neq 0\right\}$ is a non-empty open set. It was shown in [HNRR, Lemma 2] that, for $z$ in $F, \mathscr{N}_{z}$ is the linear span of the finite set $\left\{k_{w}:|w| \leq 1, \varphi(w)=\varphi(z)\right\}$.

Choose $a_{1} \in F$, and write $\left\{z:|z| \leq 1, \varphi(z)=\varphi\left(a_{1}\right)\right\}=\left\{a_{1}, a_{2}, \ldots, a_{n}\right\}$. Clearly, $\left\{a_{1}, a_{2}, \ldots, a_{n}\right\} \subset F$, and since $\varphi^{\prime}$ never vanishes on $F$, it follows from the open mapping theorem [Ru, Theorem 10.30] that there are disjoint open sets $V_{1}, V_{2}, \ldots, V_{n}$ such that

(1) $a_{k} \in V_{k}$ for $1 \leq k \leq n$,

(2) $\varphi \mid V_{1}$ is $1-1$ for $1 \leq k \leq n$,

(3) $\varphi\left(V_{1}\right)=\varphi\left(V_{2}\right)=\cdots=\varphi\left(V_{n}\right) \stackrel{\text { def }}{=} U$.

Since $\varphi\left(a_{1}\right)$ is not in $\varphi\left(D^{-} \backslash \bigcup_{k=1}^{n} V_{k}\right)$, we can assume

(4) $\varphi^{-1}(\varphi(U))=\bigcup_{k=1}^{n} V_{k}$.

For $1 \leq k \leq n$, let $\hat{f}_{k}: U \rightarrow V_{k}$ denote the inverse of $\varphi \mid V_{k}$. It follows that, for each $z$ in $U, \mathscr{N}_{z}=\operatorname{sp}\left\{k_{f_{1}(z)}, \ldots, k_{f_{n}(z)}\right\}$. Since the map $w \mapsto k_{w}$ is norm continuous on $D$, it follows that the mapping $z \mapsto P_{z}$ is norm continuous on $U$.

For each index $\lambda, B_{\lambda}^{*}$ leaves all of the $\mathscr{N}_{z}$ 's invariant, $z \in U$. Since $P_{z}$ is a continuous function of $z$, it follows that $\sigma\left(B_{\lambda}^{*} \mid \mathscr{N}_{z}\right)$ is a continuous function of $z$, with the Hausdorff metric on closed subsets of $\mathbb{C}$. It follows from the 
lemma above that, for each index $\lambda$ and each $z$ in $U, r\left(B_{\lambda}^{*} \mid \mathscr{N}_{z}\right) \leq\left\|B_{\lambda}\right\|_{e}(r$ denotes spectral radius).

However, since $B_{\lambda} \rightarrow B$ in the weak operator topology and each $\mathscr{N}_{z}$ is finite dimensional, $B_{\lambda}^{*}\left|\mathscr{N}_{z} \rightarrow B^{*}\right| \mathscr{N}_{z}$ in norm for each $z$ in $U$. It follows that $r\left(B^{*} \mid \mathscr{N}_{z}\right)=\lim r\left(B_{\lambda}^{*} \mid \mathscr{N}_{z}\right) \leq \lim \left\|B_{\lambda}\right\|_{e}=0$ for each $z$ in $U$. Since each $\mathscr{N}_{z}$ has dimension $n$, it follows that $\left(B^{*}\right)^{n} \mid \mathscr{N}_{z}=0$. Since the closed linear span of any infinite collection of $k_{w}$ 's is all of $H^{2}(\beta)$, we conclude that $B^{n}=0$.

If $p_{1}, q_{1}, p_{2}, q_{2}, \ldots, p_{m}, q_{m}$ are polynomials, and $C_{\lambda}=\sum_{k=1}^{m} p_{k}(T) B_{\lambda} q_{k}(T)$ and $C=\sum_{k=1}^{m} p_{k}(T) B q_{k}(T)$, we have $C_{\lambda} A=A C_{\lambda}$ for every $\lambda, C_{\lambda} \rightarrow C$ in the weak operator topology, and $\left\|C_{\lambda}\right\|_{e} \rightarrow 0$. We can apply the above arguments to get that $C^{n}=0$.

We can now follow the arguments in [HNRR, p. 413] to conclude that $B=$ 0 . More precisely, suppose $z \in U$ and $\left\{k_{w_{1}}, \ldots, k_{w_{n}}\right\}$ is the basis for $\mathscr{N}_{z}$, and, for $1 \leq i \leq n$, let $P_{i}$ denote the projection of $\mathscr{N}_{z}$ onto $k_{w_{i}}$ parallel to $\operatorname{sp}\left\{k_{w_{j}}: j \neq i\right\}$. Since $T^{*} \mid \mathscr{N}_{z}$ is diagonal with distinct eigenvalues, we have that each $P_{i}$ has the form $p_{i}(T)^{*} \mid \mathcal{N}_{z}$ for some polynomial $p_{i}$. Hence, for any $n \times n$ matrix $\left(b_{i j}\right)$, the Schur product of $\left(b_{i j}\right)$ with $B^{*} \mid \mathscr{N}_{z}$ is nilpotent, since it equals $C^{*} \mid \mathscr{N}_{z}$, where $C=\sum_{i, j=1}^{n} \bar{b}_{i j} p_{j}(T) B p_{i}(T)$ has the form of the preceding paragraph. It follows from [HNRR, Lemma 1] that $B^{*} k_{w_{j}}=0$ for some $j, 1 \leq j \leq n$. Thus $B^{*} k_{w}=0$ for infinitely many $w$, so $B^{*}=0$.

We should point out that what we have referred to as "Lomonosov's hypothesis" is only a special case of Lomonosov's powerful results. Even the original theorem can be phrased in such a way so as to include every operator; namely:

An operator has a non-trivial invariant subspace if and only if it is contained in a proper weakly closed algebra that contains a non-zero compact operator.

The reverse implication follows from the fact that if $M$ is a non-trivial subspace, then $\operatorname{Alg}(M)=\{T: T(M) \subset M\}$ is a proper weakly closed algebra containing a non-zero compact operator.

\section{ACKNOWLEDGMENTS}

The author gratefully acknowledges support from the National Science Foundation while this research was undertaken. The author would also like to thank Victor Lomonosov for asking the question addressed by this paper.

\section{REFERENCES}

[C] C. Cowen, An analytic Toeplitz operator that commutes with a compact operator, J. Funct. Anal. 36 (1980), 169-184.

[HNRR] D. Hadwin, E. Nordgren, H. Radjavi, and P. Rosenthal, An operator not satisfying Lomonosov's hypothesis, J. Funct. Anal 38 (1980), 410-415.

[L1] V. Lomonosov, Invariant subspaces for operators commuting with compact operators, Funkcional. Anal. i Priložen 7 (1973), 55-56; English transl., Functional Anal. Appl. 7 (1973), 213-214.

[L2] _ An extension of Burnside's theorem to infinite-dimensional spaces, Israel J. Math. 75 (1991), 329-339.

[Ru] W. Rudin, Real and complex analysis, McGraw-Hill, New York, 1987.

Department of Mathematics, University of New Hampshire, Durham, New Hampshire 03824

E-mail address: don@math.unh.edu 